\title{
The Bounds on Tracking Performance Utilising a Laser-Based Linear and Angular Sensing and Measurement Methodology for Micro/Nano Manipulation
}

\author{
Leon Clark ${ }^{\mathrm{a}, 1}$, Bijan Shirinzadeh ${ }^{\mathrm{a}}$, Yanling Tian ${ }^{\mathrm{b}}$, and Yongmin Zhong ${ }^{\mathrm{c}}$ \\ ${ }^{a}$ Robotics and Mechatronics Research Laboratory, Department of Mechanical and Aerospace Engineering, \\ Monash University, Clayton, VIC 3800, Australia \\ ${ }^{b}$ Key Laboratory of Mechanism Theory and Equipment Design of Ministry of Education, Tianjin University, \\ Tianjin 30072, China \\ ${ }^{c}$ School of Aerospace, Mechanical and Manufacturing Engineering, RMIT University, Bundoora, VIC 3083, \\ Australia
}

\begin{abstract}
This paper presents an analysis of the tracking performance of a planar three Degrees of Freedom (DOF) flexure-based mechanism for micro/nano manipulation, utilising a tracking methodology for the measurement of coupled linear and angular motions. The methodology permits trajectories over a workspace with large angular range through the reduction of geometric errors. However, when combining this methodology with feedback control systems, the accuracy of performed manipulations can only be stated within the bounds of the uncertainties in measurement. The dominant sources of error and uncertainty within each sensing subsystem are therefore identified, which leads to a formulation of the measurement uncertainty in the final system outputs, in addition to methods of reducing their magnitude. Specific attention is paid to the analysis of the vision-based subsystem utilised for the measurement of angular displacement. Furthermore, a feedback control scheme is employed to minimise tracking errors, and the coupling of certain measurement errors is shown to have a detrimental effect on the controller operation. The combination of controller tracking errors and measurement uncertainty provides the bounds on the final tracking performance.
\end{abstract}

Keywords: measurement error and uncertainty, micro/nano manipulation, feedback control, visionbased angular measurement

\section{Introduction}

The ability to perform fast, precise and accurate manipulations at the micro/nano scale has been key to many developments in engineering and scientific fields, particularly in recent advancements in

\footnotetext{
${ }^{1}$ Corresponding author. Email: leon.clark@monash.edu

This is a post-print version of: L. Clark, B. Shirinzadeh, Y. Tian, and Y. Zhong. The bounds on tracking performance utilising a laser-based linear and angular sensing and measurement methodology for micro/nano manipulation. Measurement Science and Technology, 25(12):125005, 2014, DOI: $10.1088 / 0957-0233 / 25 / 12 / 125005$.
} 
nanotechnology. Mechanisms which are capable of producing such motions play an important enabling role in present and future technologies including scanning probe microscopy, the grasping of miniature objects, micro-component manufacture and assembly, cell manipulation and optical steering [1-6].

Recent research into micro/nano manipulation systems has led to the development of many mechanisms with varying Degrees of Freedom (DOF). In particular, focus has been dedicated to positioners producing linear motion [7-9]. However, studies have been conducted to investigate mechanisms with additional axes, hence introducing rotational motion [10,11]. Wire Electrical Discharge Machining (WEDM), commonly used to manufacture these mechanisms, often limits designs to lie within a single plane. As such, many manipulation stages have been produced which perform planar motions with three DOFs - in the $X, Y$ and $\theta$ axes.

Typically, manipulation tasks are performed within the range between several hundred micrometres to fractions of a nanometre, and recent developments demand higher performance in terms of accuracy and response speed. A set of features common to most systems has been adopted to satisfy these requirements. Compliant elements such as flexure hinges and cantilevers are often employed in the design of such mechanisms, which replace revolute joints that can suffer from backlash, stick-slip friction and other related effects. These elements act as bearings permitting continuous, smooth motion. They can be manufactured monolithically preventing any assembly errors, utilising various geometries to customise rotational precision and equivalent stiffness [12]. Actuation of the mechanism is often provided by piezoelectric actuators (PEAs), which exhibit high stiffness, fast response and can provide a displacement input with infinite resolution.

However, the response of a PEA to a voltage input has significant hysteresis, as well as other non-linear effects such as drift. Hence, the requirement for accurate tracking of desired end effector motion has mandated the use of control techniques. Previous studies have proposed the use of various feedback control methodologies to improve tracking performance [13-16]. Furthermore, feedforward techniques, such as those utilising an inverse model of the PEA hysteresis in combination with feedback control have also been proposed [17-19].

Measurement of the motion of the manipulator is therefore important for both feedback and verification. Capacitive and laser interferometer-based sensing and measurement techniques have gained favour for translational motion due to their fast response and high resolution. Similarly, high resolution image sensors have also been utilised for inspection and manipulation at small scales [20,21]. Of these, laser interferometer-based methods are very promising as they function over a practically unlimited range, and have been shown to be suitable for feedback control of linear positioners [22]. However, rotational motion introduces misalignment, and thus geometric errors, and necessitates alternative methodologies for the determination of the end effector pose. For this reason, previous studies investigating control of 


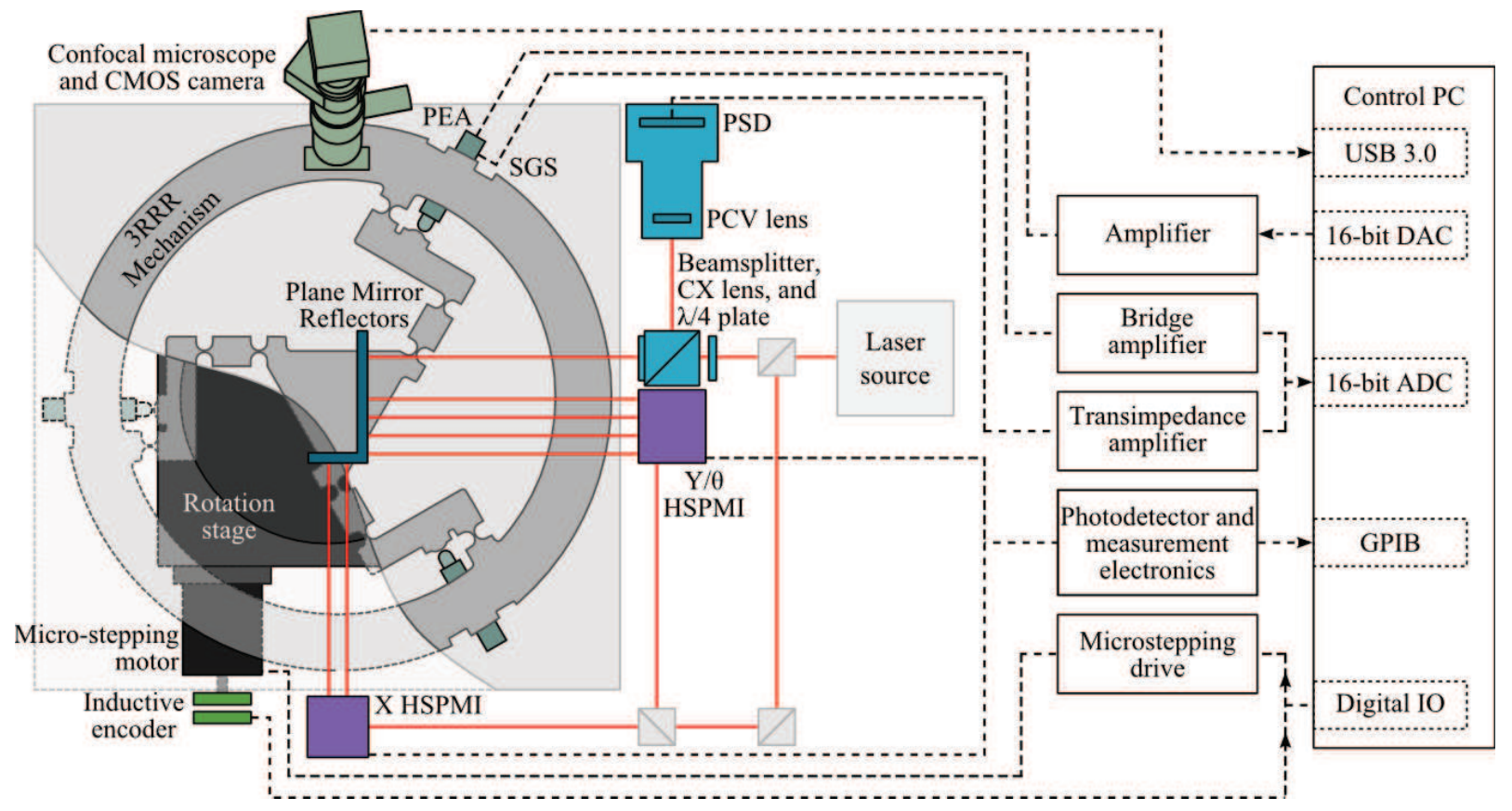

Fig. 1: Schematic of the experimental apparatus.

flexure mechanisms which perform angular motions have considered any rotation to be undesirable, and employed feedback control for its reduction [10, 23-25].

In previous research, the authors have proposed a novel tracking technique to provide position measurement of coupled linear and angular motions [26]. In implementing such a technique, pose output is determined from the combination of measurements from multiple sub-systems. Hence, any uncertainty in the final output will be an accumulation of the individual uncertainties of each component. These may have contributions from random variations due to vibrations and noise, as well as other fluctuations in the environment. Similarly, errors may result from sensor inaccuracies, possibly due to geometric errors, and poor calibration. Identification and analysis of each source of error and uncertainty is therefore vital to both investigate the limitations of such a system and their subsequent impact on control performance, and develop means for calibration and uncertainty minimisation.

\section{Pose Measurement Methodology}

A tracking methodology has been proposed for the measurement of three DOF planar motion in previous research [26]. It utilises a laser interferometry-based sensing technique for measurement of motion for the two linear axes, and a vision-based scheme for the angular axis. The methodology employs a rotation stage beneath the mechanism's base to provide a counter-rotation in response to any misalignment introduced by $\theta$ motion. In this way, geometric errors within the laser-based measurement system are minimised. A schematic of the apparatus is shown in Fig. 1. 


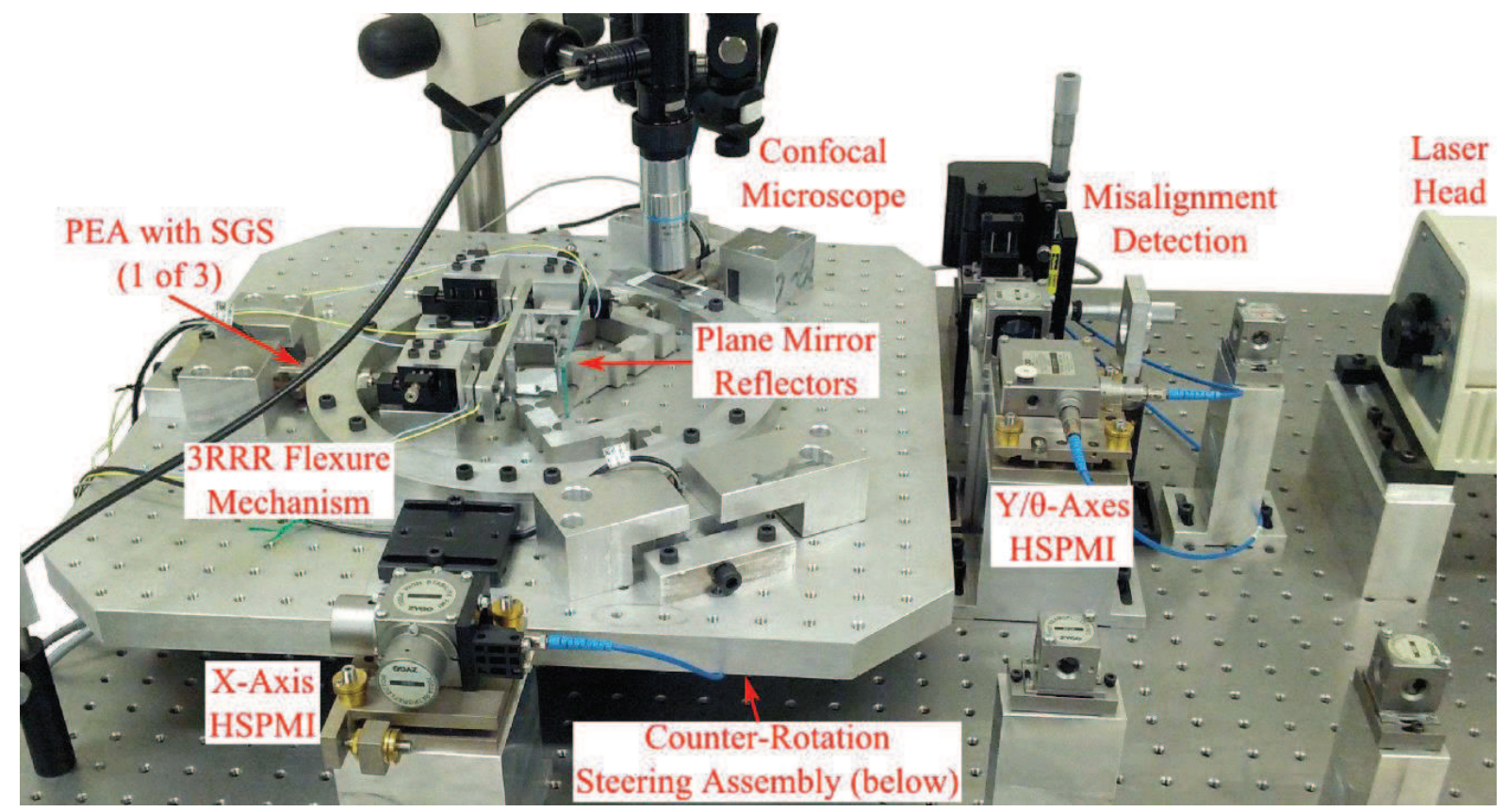

Fig. 2: Photograph of the experimental apparatus.

\subsection{Experimental Apparatus}

Experimentation is performed to examine the positioning control and tracking of a flexure-based mechanism, which is capable of producing planar motions with three DOFs. The steering assembly which provides the counter-rotation consists of a precision rotation stage actuated by a micro-stepping drive. A rotational resolution of $2.74 \mu \mathrm{rad}$ is achievable in a single step. Attached to the shaft of the motor is an inductive angle encoder possessing a resolution of $2.13 \mu \mathrm{rad}$. As hysteresis is observed between the inductive encoder output and the actual stage rotation, the measurement of the base's rotation, $\theta_{V}$, is performed directly with a vision-based technique, to be described in Section 2.2.Misalignment of the measurement beams, $\theta_{e}$, is detected by a Position Sensitive Diode (PSD) based detection unit, as described in [26]. The misalignment sensor employs a plano-concave (PCV) lens to amplify the deflection of the measurement beam, together with a convex (CX) lens to ensure a high signal-to-noise ratio. Two High-Stability Plane Mirror Interferometers (HSPMI) are mounted in the directions of the $X$ and $Y$ axes. The $X$ axis HSPMI records only linear motion. The HSPMI unit in the $Y$ direction also provides a redundant yaw axis, which is instead measured by the misalignment detection system. The rate of position acquisition is limited by the speed of the GPIB bus which provides $\left(x_{i}, y_{i}\right)$ coordinate pairs to the control computer at $95 \mathrm{~Hz}$.

The developed mechanism employs the 3-RRR parallel kinematic chain, and is a monolithic flexurebased mechanism manufactured using WEDM from aluminium alloy 7075-T6 [27]. The mechanism is driven by three PEAs, each with an embedded Strain Gauge Sensor (SGS), capable of delivering a maximum $60 \mu \mathrm{m}$ displacement at $100 \mathrm{~V}$. A photograph of the experimental setup is shown in Fig. 2. 


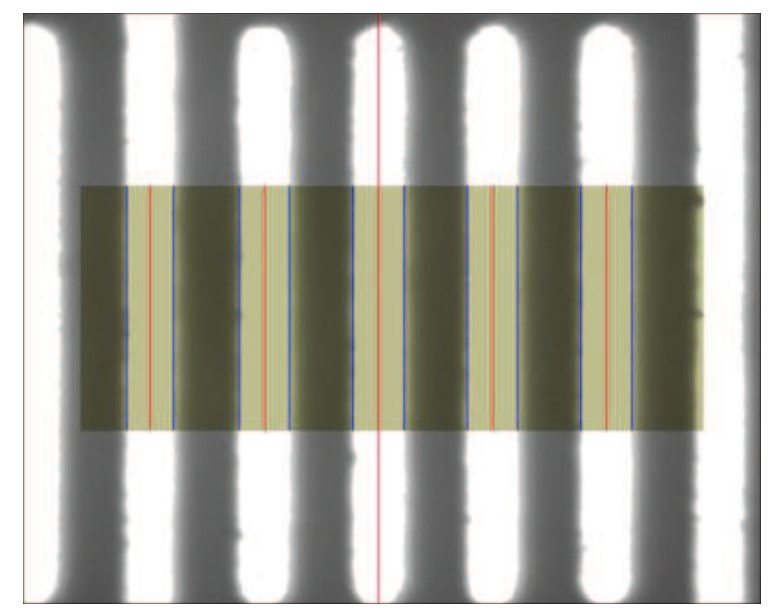

Fig. 3: Vision-based technique used for angular measurement. The vertical lines indicate the tracked rulings, whilst the greyed window indicates the region utilised for computations.

A computer using a real-time extension performs the data acquisition and analysis, control computations, and actuation tasks. This computer utilises 16-bit digital-to-analogue and analogue-todigital converters.

Control tasks, detailed in Section 3, are performed at $1 \mathrm{kHz}$ (inner loop) or $90.9 \mathrm{~Hz}$ (main loop and misalignment controller), whilst commands to the actuator for the steering assembly and measurement of the PSD voltage are performed at $25 \mathrm{kHz}$. Whilst higher control frequencies are typically utilised, for the purposes of this study, the main loop is run at a relatively slow rate. This will allow the magnitude of data age uncertainties on both measurement and tracking to be more readily observed.

\subsection{Vision-based Angular Measurement Methodology}

The vision-based measurement of rotation employs a confocal microscope to view the displacement of a micrometre scale calibration slide, and functions in a similar fashion to a linear encoder. The slide, with vertical rulings spaced every $10 \mu \mathrm{m}$, is placed a fixed radius from the rotation centre on the mechanism's base. The methodology measures the displacement of the mechanism's base during counter-rotation relative to the fixed microscope.

Upon acquisition of a frame, pixels within a chosen window are averaged vertically, and the edges of each ruling are detected. This averaging reduces the effects of artefacts at the boundaries of the rulings. Furthermore, as only a central window in considered, the algorithm is less sensitive to image distortion and uneven illumination away from the image centre. The centreline of each of the rulings can then be calculated from its edges, which provide features for both tracking and the determination of the image scale (in microns per pixel). As the image scale can vary, this method tolerates out-of-plane movement of the slide. The centremost ruling is tracked between frames, and the cumulative displacement is interpreted as the arc-length traversed as the stage rotates. As only the central ruling is tracked, and may change at any time, the technique permits the measurement of angles far greater than those limited by 


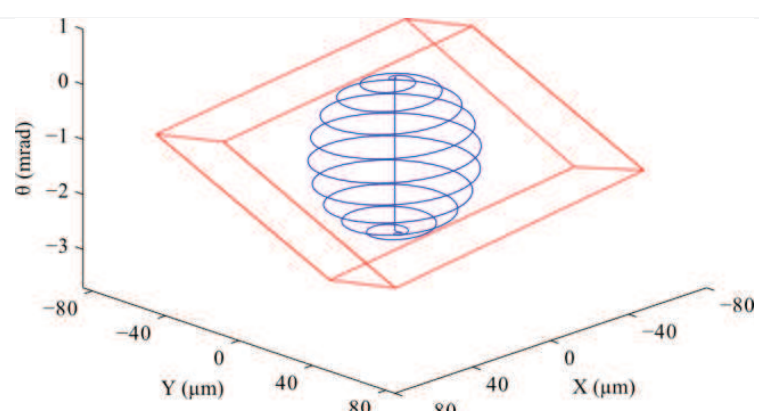

(a)

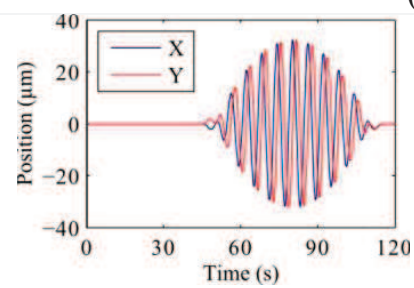

(b)

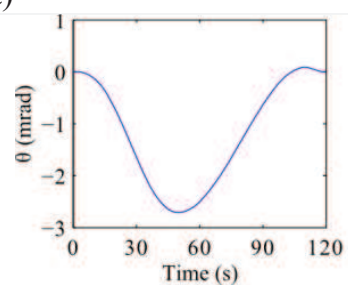

(c)

Fig. 4: Desired three DOF jerk-free spiral trajectory: (a) 3D view shown within mechanism workspace, (b) X and Y trajectories, (c) $\theta$ trajectory.

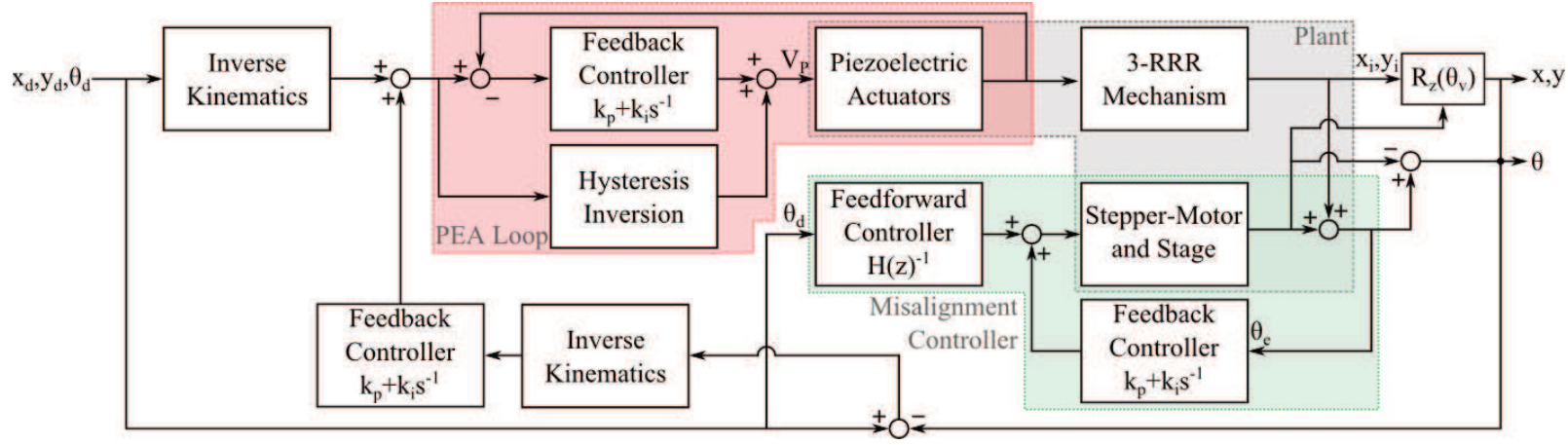

Fig. 5: Schematic of the proposed control methodology.

the width of the frame. Furthermore, the resolution is only limited by the capabilities of the microscope and the ruling spacing of the calibration slide.

A high-speed CMOS sensor has been utilised, where $1280 \times 1024$ pixel images are processed at approximately 135 frames per second. Fig. 3 shows a processed frame, where the ruling edges and centres have been identified by the algorithm. The centremost ruling for that particular frame is also indicated. Further details relating to the implementation of the technique can be found in [28].

\subsection{Test Trajectory}

The trajectory utilised for experimental testing is a three DOF spiral trajectory within the workspace of the mechanism as shown in Fig. 4. The trajectory consists of three phases: an initial rotation to the counter-clockwise limit, the spiral section, and a rotation back to the origin. During the middle phase, the $X$ and $Y$ components consist of sinusoids whose amplitudes are modulated within a parabolic envelope. All other sections are constructed from polynomials to ensure continuity of the position, velocity and acceleration. 


\section{Combined Manipulation and Misalignment Control Methodology}

Fig. 5 shows an overview of the control methodology proposed to permit the tracking of desired trajectories with three DOFs, whilst minimising angular misalignment within the laser-based measurement system. The misalignment component of the controller provides the input to the steering assembly, and hence works to counter-rotate the mechanism base to eliminate the misalignment of the laser-beam [26].

The manipulation component provides inputs to the PEAs, and controls the positioning of the 3-RRR mechanism's stage. It is proposed that this control scheme consists of inner and outer loops. The inner loop works to linearise the input-output response of each PEA. It consists of a feedforward component, which is an inverse model of the hysteresis of each actuator. The feedback component (a PI controller), using SGS measurements, acts to eliminate any discrepancy between the desired and actual outputs, due to disturbances or errors in the hysteresis model.

The main outer loop controls the actual positioning of the mechanism, and passes the desired PEA displacements to the inner loop. This utilises the inverse kinematics to provide a feedforward component, summed with the output of a feedback PI controller which utilises the combined measurements from the PSD, laser interferometer-based, and vision-based systems.

\subsection{Inverse Kinematics}

A linearised inverse kinematic relationship for the stage has been derived. For flexure mechanisms driven by PEAs, the small range of motion of each joint is such that a linear approximation of the kinematics is accurate [29]. This can be performed through a linearisation of the full analytic equations, or through geometric techniques such as loop-closure [30,31].

In this study an experimentally determined Jacobian has been utilised. It was found through a leastsquares fitting of the Jacobian to experimental data from a large set of points within the workspace of the mechanism. The linear inverse kinematic relationships were found as:

$$
\left[\begin{array}{l}
p_{1} \\
p_{2} \\
p_{3}
\end{array}\right]=J\left[\begin{array}{l}
x \\
y \\
\theta
\end{array}\right]=\left[\begin{array}{ccc}
0.3468 & -0.1913 & -0.0113 \\
0.0004 & 0.3857 & -0.0107 \\
-0.3246 & -0.1885 & -0.0107
\end{array}\right]\left[\begin{array}{l}
x \\
y \\
\theta
\end{array}\right]
$$

This can be compared with the analytic linearised Jacobian, found through the loop-closure technique, given below in (2). The experimentally determined Jacobian will be more accurate as it accounts for the different behaviour of each PEA (due in part to differing PEA preloads) and imperfections in the manufacturing processes for the mechanism.

$$
J_{a}=\left[\begin{array}{ccc}
0.3360 & -0.1940 & -0.0097 \\
0 & 0.3880 & -0.0097 \\
-0.3360 & -0.1940 & -0.0097
\end{array}\right]
$$




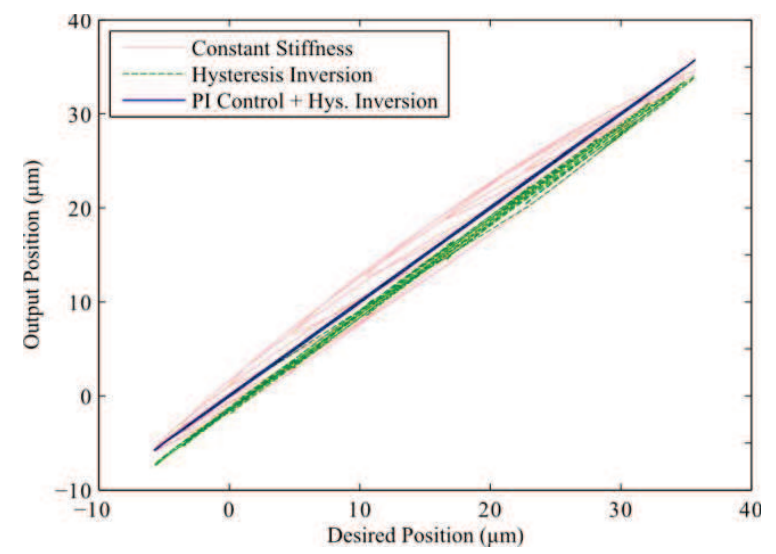

Fig. 6: Performance of the inner loop controller during experimentation.

\subsection{Inner Loop}

The inner loop of the controller acts to linearise the input-output position relationship of each PEA. It consists of feedforward and feedback components. The feedforward component utilises a rateindependent inverse Prandtl-Ishlinskii model of the non-linear hysteresis of the PEA. The modelling has been performed directly from the experimental data, utilising a least-squares optimisation procedure for parameter identification, as outlined in [32]. A PI controller has been utilised as the feedback controller, deriving its error signal from the measurement provided by each SGS. The output of the first PEA is shown in Fig. 6, for the cases where the PEA is considered to have a constant stiffness (in $\mathrm{V} / \mathrm{m}$ ), with the feedforward hysteresis inversion, and with the addition of PI control. It can be seen that the hysteresis inversion adequately linearises the output. However, an offset error accumulates during the initial loading, which is minimised through feedback control.

The inner loop is run at $1 \mathrm{kHz}$, which is eleven times faster than the main loop. This ensures that the availability of high-speed data from each SGS is appropriately utilised. Due to this difference in control speed, the input to the inner loop is interpolated linearly over the length of the outer-loop period, so that the desired position is achieved just before the next outer-loop computation.

\section{Analysis of Uncertainties}

The sensor outputs not only constitute the feedback controller input, but also the final output of the entire manipulator. Ultimately, successful completion of a positioning task is dictated by the quality of the outputs from the measurement system. However, measurement by any sensing technique only provides an estimate of the value of the measurand. Accompanying any measurement output is an associated uncertainty, as well as any offsets or other calibration errors.

Identification of the bounds of errors and uncertainties is therefore important to establish the limits of tracking performance. In the case of the measurement apparatus presented in this paper, the final $X, Y$ and $\theta$ outputs of the system are calculated from multiple sources. Hence, uncertainties in each measurement input will propagate to the outputs. The knowledge of errors from each sensing 


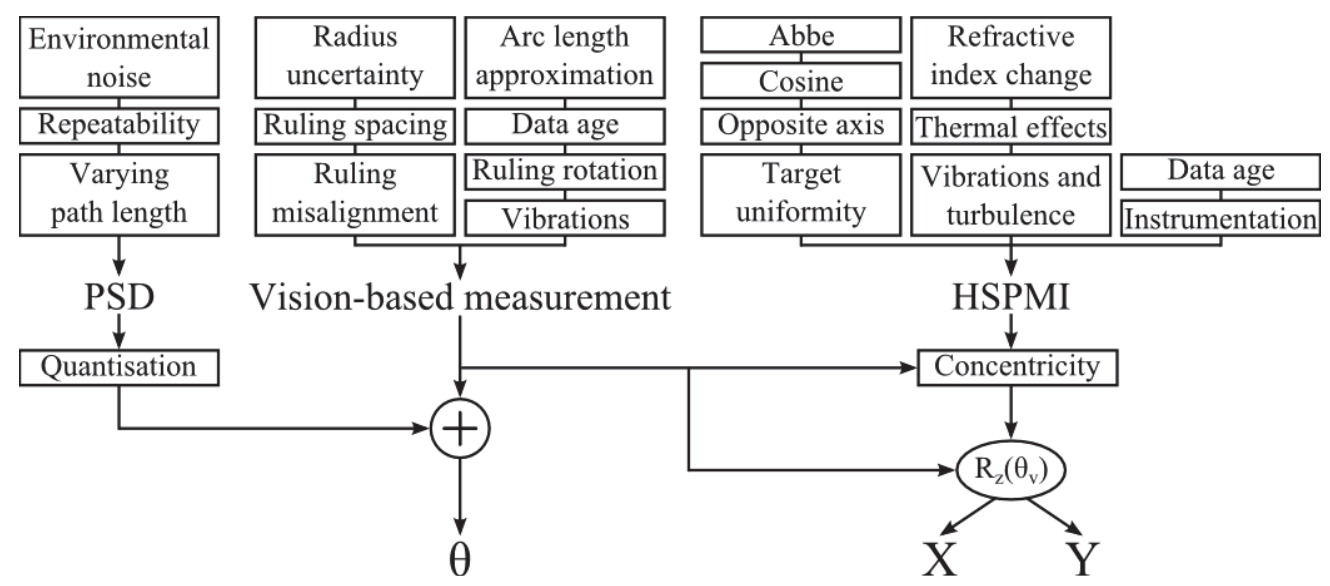

Fig. 7: Propagation of sources of uncertainty to the final outputs.

component provides potential means of measurement correction. The detailed analysis and estimation of these processes form the subject of this section.

The measurement methodology under investigation in this paper draws information from three different sensor techniques: the PSD-based misalignment sensor, laser interferometry-based displacement measurement, and vision-based angular measurement. Whilst the SGSs are also utilised for feedback, this section focusses on only the final output of the measurement system, being the $X, Y$ and $\theta$ positions. Fig. 7 shows a graphical representation of the progression of input uncertainties to each output.

\subsection{Misalignment sensor}

This sub-system consists of the PSD-based measurement device, along with the reflector and optical components which provide the amplification of the laser-beams deflection. The thin lens equation predicts the deflection, $d_{\mathrm{PSD}}$, of the laser beam at the $\mathrm{PSD}$, due to a rotation $\theta_{e}$ of the target mirror, to be:

$$
d_{\mathrm{PSD}}=\frac{R|f|+L(R-L)}{|f|} \tan \theta_{e}
$$

where $f$ is the focal length of the PCV lens, $R$ is the total optical path length from the reflector to the PSD, and $L$ is the distance between the PCV lens and the PSD. This position is converted to an output voltage by measurement electronics which produce the quotient of the difference and sum of the photocurrents at the terminals of the PSD (converted to a voltage using a transimpendence amplifier). In effect, for small angles, the output voltage is directly proportional to the misalignment angle.

The primary use of (3) is for sensor design, as the PSD's output has been experimentally calibrated against the yaw axis of the HSPMI. However, this was performed whilst the reflector was simply rotated, without coupled translation. When the flexure stage is actuated, the optical path length $R$ will change. The fractional change in output can be approximated as: 
Table 1: Estimation of Maximum PSD Errors and Uncertainties

\begin{tabular}{cccc}
\hline \hline & Source & Governing Equation & Value \\
\hline Errors & Varying path length & $(4),(6)$ & $0.5 \mathrm{nrad}$ \\
\hline \multirow{3}{*}{ Uncertainties } & Environmental Noise & & $0.30 \mu \mathrm{rad}$ \\
& Repeatability & & $0.6 \mu \mathrm{rad}$ \\
& Quantisation & $0.03 \mu \mathrm{rad}$ \\
\hline \hline
\end{tabular}

nb. The magnitude of uncertainties is given as the maximum standard uncertainty.

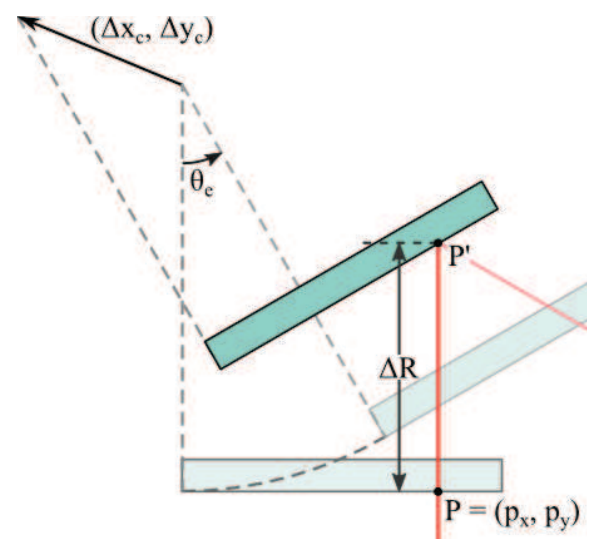

Fig. 8: Change in optical path length due to motion of stage.

$$
\frac{\Delta \theta_{e, R}}{\theta_{e}}=\frac{\Delta d_{\mathrm{PSD}}}{d_{\mathrm{PSD}}}=\frac{\Delta R}{R}
$$

A situation giving rise to a change in path length is shown in Fig. 8, where $P$ is the original position of the point of reflection, which is offset from the rotational centre. When the reflector is displaced by thevector $\left(\Delta x_{c}, \Delta y_{c}\right)$ and a misalignment error $\theta_{e}$ exists, the intersection of the reflector and the laser beam at $P^{\prime}$ allows the change in path length (the difference in vertical position) to be found as:

$$
\Delta R=p_{y}\left(\cos \theta_{e}-1+\sin \theta_{e} \tan \theta_{e}\right)+p_{x} \tan \theta_{e}+\Delta y_{c}-\Delta x_{c} \tan \theta_{e}
$$

Furthermore, due to the counter-rotation process, $\left(\Delta x_{c}, \Delta y_{c}\right)$ is the rotation of the vector $(x, y)$ by the angle $-\theta$. This can be combined with (5) to determine the total change in path length. To first order in $\theta$ and $\theta_{e}$, this is:

$$
\Delta R \approx p_{x} \theta_{e}+x \theta-x \theta_{e}+y
$$

Sources of uncertainty include the quantisation error of the 16-bit analogue-to-digital converter, and the repeatability of the sensor, in addition to the noise within the sensor output. Repeatability has been measured by repeatedly rotating the stage, and finding the standard deviation of the PSD output for a given HSPMI yaw measurement. A summary of the maximum magnitudes of each error and uncertainty source during the trajectory is listed in Table 1. 
Table 2: Estimation of Maximum HSPMI Uncertainties

\begin{tabular}{ccc}
\hline \hline Source & Governing Equation & Standard Value \\
\hline Abbe & $(9)$ & $2 \mathrm{~nm}(X), 58 \mathrm{~nm}(Y)$ \\
Cosine & $(8)$ & $\sim 0$ \\
Opposite axis & $(7)$ & $\sim 0$ \\
Target uniformity & & $18.3 \mathrm{~nm}$ \\
\hline Refractive index change & & $18.2 \mathrm{~nm}$ \\
Thermal expansion & & $0.5 \mathrm{~nm}$ \\
Vibrations and turbulence & & $25 \mathrm{~nm}$ \\
\hline Data age & $(10)$ & $104 \mathrm{~nm}$ \\
Instrumentation & & $2.6 \mathrm{~nm}$ \\
\hline \hline
\end{tabular}

\subsection{Laser Interferometry-based Sensing and Measurement}

The laser interferometry-based sensing and measurement technique employs HSPMI units as detailed in Section 2.1. These are heterodyne interferometers which measure displacement through the beat frequency between two signals spaced $20 \mathrm{MHz}$ apart. Uncertainties in interferometer measurement can be divided into three categories: geometric, environmental and instrumental. A summary of each of the identified uncertainty sources within the HSPMI system is provided in Table 2.

\subsubsection{Geometric Sources}

Geometric errors and uncertainties arise through imperfections in the setup of the target mirror. Each of these sources is depicted in Fig. 9.

For this apparatus, the $X$ and $Y$ axes are defined to be parallel with the axes of the interferometer setup. Hence when $\theta_{e}$ is zero and the PEAs are at their preload voltages, the tool centre point lies at the origin. Due to this configuration, standard cosine (I) errors as shown in Fig. 9a are eliminated. However, they may still be present if the interferometer axes are not perfectly orthogonal. If the $X$ measurement axis is misaligned by an angle $\phi$ relative to a line perpendicular to the $Y$ measurement axis, the opposite axis uncertainty in measurement will be:

$$
\Delta x_{i, \mathrm{opp}}=x_{i}(1-\cos \phi) \approx \frac{1}{2} x_{i} \phi^{2}
$$

Careful beam alignment procedures have been undertaken during the initial interferometer setup, and it is expected that any orthogonality between the axes would be less than $0.1^{\circ}$. Hence, this uncertainty will be negligible due to the quadratic dependence on the misalignment.

The counter-rotation methodology was established to minimise the misalignment of the laser beam. As a result, cosine (II) errors (Fig. 9b) are effectively eliminated. The magnitude of this cosine error is determined by the following relationship:

$$
\Delta x_{i, \cos }=a\left(\sec 2 \theta_{e}-1\right) \approx 2 a \theta_{e}^{2}
$$




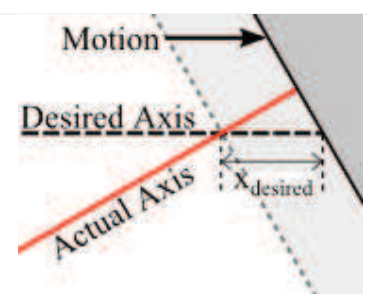

(a)

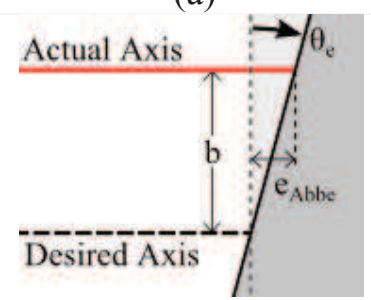

(c)

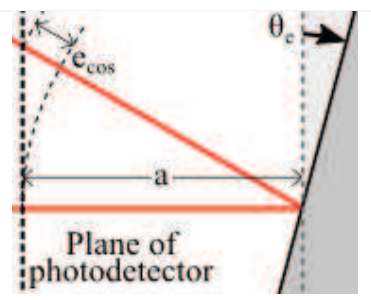

(b)

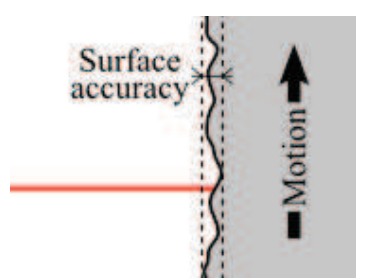

(d)

Fig. 9: Geometric errors within HSPMI measurements: (a) Cosine error (I), (b) Cosine error (II), (c) Abbe error, (d) Target uniformity error.

With the misalignment minimised using the counter-rotation methodology, $\theta_{e}$ is reduced to a small value and varies quickly. Whilst it is possible in principle to correct for this error and Abbe errors, this cannot be performed accurately. Hence, these are considered to be uncertain. For misalignments kept within $2 \mu \mathrm{rad}$ (standard uncertainty), this cosine uncertainty is of the order of $10^{-12} \mathrm{~m}$.

The Abbe uncertainty arises due to the measurement axes of the interferometer being laterally misaligned with the desired axis, as depicted in Fig. 9c. Ideally, these desired axes pass through the centre of the positioning stage. However, due to the size of the interferometer, and the misalignment measurement beam sharing the same mirror, there is a $30 \mathrm{~mm}$ distance separating the HSPMI axis from the nominal $Y$ axis. The magnitude of the Abbe uncertainty is found as:

$$
\Delta x_{i, \mathrm{Abbe}}=b \tan \theta_{e}
$$

The final source of geometric uncertainty results from non-uniformity in the target mirror, as shown in Fig. $9 \mathrm{~d}$. This will be caused by variations in the surface profile of the target mirror. For the mirrors utilised $(\lambda / 10$ surface accuracy), the standard uncertainty is $18.3 \mathrm{~nm}$.

\subsubsection{Environmental Sources}

Changes in the environment have the effect of changing the refractive index of the air path. These include changes in temperature, pressure and humidity. Edlen's equation was used to calculate the refractive index before commencing experimentation, to account for the experimental conditions deviating from Standard Temperature and Pressure (STP) [33]. Following this, during experimentation changes in the environmental conditions were kept within $0.1{ }^{\circ} \mathrm{C}$ in temperature, $0.15 \mathrm{mmHg}$ in pressure and 5\% in humidity (assumed to be uniformly distributed within these intervals). Following the manufacturer's specifications, the standard uncertainty due to the change in refractive index has been 
Table 3: Estimation of Maximum Vision-based Measurement Errors and Uncertainties

\begin{tabular}{cccc}
\hline \hline \multirow{2}{*}{ Errors } & Source & Governing Equation & Value \\
\hline \multirow{6}{*}{ Arc-length approximation } & $(15)$ & $6.6 \mathrm{nrad}$ \\
& Ruling rotation & $(17)$ & $3.3 \mathrm{nrad}$ \\
\hline Dncertainties & Data age & & $0.71 \mu \mathrm{rad}$ \\
& Image distortion & $(10)$ & $1.2 \mu \mathrm{rad}$ \\
& Radius variation & & $\sim 0$ \\
& Ruling edge artefacts & $(13)$ & $0.56 \mu \mathrm{rad}$ \\
& Ruling misalignment & $(14)$ & $0.05 \mu \mathrm{rad}$ \\
& Ruling spacing & $(13)$ & $0.017 \mu \mathrm{rad}$ \\
& Slide tilting/pitching & $(20)$ & $0.034 \mu \mathrm{rad}$ \\
& Scale variation and pixel size & & $0.11 \mu \mathrm{rad}$ \\
\hline Vibration and environmental noise & &
\end{tabular}

estimated to be $18.2 \mathrm{~nm}$. Temperature changes also cause the expansion or contraction of components within the interferometer. The thermal drift coefficient is given as $0.018 \mu \mathrm{m} /{ }^{\circ} \mathrm{C}$.

The largest sources of environmental uncertainty are due to vibrations and air turbulence. The magnitudes of these uncertainties have been estimated as the RMS noise whilst the mirror was held stationary.

\subsubsection{Instrumental Sources}

Instrumentation errors are due to imperfections in the construction of the optical system. This includes the mixing of the two polarisation components within the laser beam, instability of the laser frequency, any inaccuracies of the processing electronics, and the underlying resolution of the measurement process. These are dependent upon the specifications of the optics and electronics, which are provided by the manufacturer.The other source is due to uncertainty in the age of the data returned by the sensor. The minimum age of the data produced by the measurement electronics is $10 \mathrm{~ns}$. Due to the limited transfer speed of the interferometer-to-computer bus (GPIB), the standard uncertainty in data age is $3.1 \mathrm{~ms}$. This uncertainty (given as a distance) can be estimated by (10).

$$
\Delta x_{i, \text { age }}=\frac{d x_{i}}{d t} \Delta t_{\mathrm{age}}
$$

\subsection{Vision-based Measurement Technique}

Errors within the vision-based measurement technique can be divided to those resulting from the experimental setup, and those occurring during experimentation. An estimation of the maximum magnitude of each source during the trajectory is given in Table 3 . 


\subsubsection{Errors due to Experimental Setup}

The linear displacement $d_{V}$ is approximately the arc-length displaced at a radius from the rotation centre of $R_{V}$, permitting the arc-length approximation, (11), to be used to calculate $\theta_{V}$. Hence, uncertainty in the radius will induce uncertainty in the final output.

$$
\theta_{V}=\arctan \frac{d_{V}}{R_{V}} \approx \frac{d_{V}}{R_{V}}
$$

In practice, there would be uncertainty in this radius due to the manufacturing tolerances on the features used to locate the calibration slide. However, in this study the vision-based system has been calibrated against the interferometer, producing a better estimate of $R_{V}$. However, the radius may still vary due to incorrect placement of the slide and concentricity (which is discussed in Section 4.4). It has been assumed that the standard deviation of the radius is $0.1 \mathrm{~mm}$. Due to (11), the fractional uncertainties of the radius and the output angle can be equated:

$$
\frac{\Delta \theta_{V, R_{V}}}{\theta_{V}}=\frac{\Delta R_{V}}{R_{V}}
$$

The initial misalignment of the rulings on the calibration slide, where the rulings are not truly vertical, causes the scale to be overestimated. For a misalignment of $\gamma$, this leads to uncertainty in the image scale, and hence an uncertainty in $\theta_{V}$ of:

$$
\Delta \theta_{V, \text { misalign }}=\theta_{V}(\sec \gamma-1) \approx \frac{1}{2} \theta_{V} \gamma^{2}
$$

A standard uncertainty of $0.5^{\circ}$ has been assumed for this misalignment.

It is also possible that the normal to the calibration slide is tilted with respect to the yaw axis, causing the image scale to be underestimated. Due to averaging of the pixels in the direction perpendicular to the slide's translation, only pitching in the direction of travel needs to be considered. Assuming the pitch remains approximately constant during motion, (13) can again be used to estimate this uncertainty. Due to the manufacturing tolerances on the flexure mechanism, a standard uncertainty in pitch of $0.2^{\circ}$ has been assumed.

The rulings on the slide will themselves have uncertain positions. The overall accuracy, $d_{\mathrm{acc}}$, of any measurement taken by the slide over its full length is specified to be less than $1 \mu \mathrm{m}$ (assumed uniformly distributed). Assuming that this error increases linearly from the initial point of measurement, becoming a maximum at the full length $\left(L_{\text {slide }}\right)$ of the slide, the uncertainty can be estimated by the following:

$$
\Delta \theta_{V, \text { ruling }}=\frac{d_{\text {acc }}}{L_{\text {slide }}} \theta_{V}
$$

Errors may also be caused by distortion within the optical system. In the case of the experimental apparatus used for this study, the microscope utilised a apochromat objective lens with flat-field (plan) 


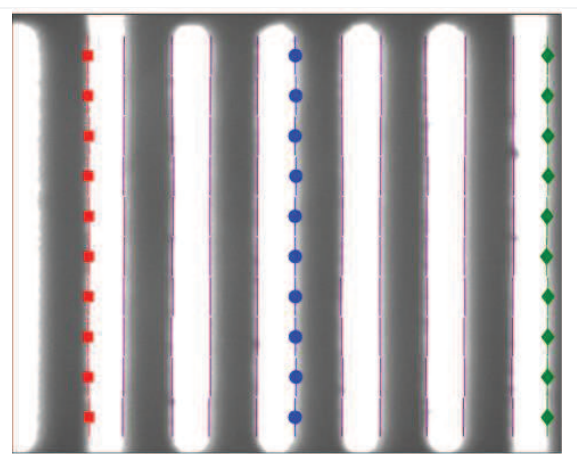

(a)

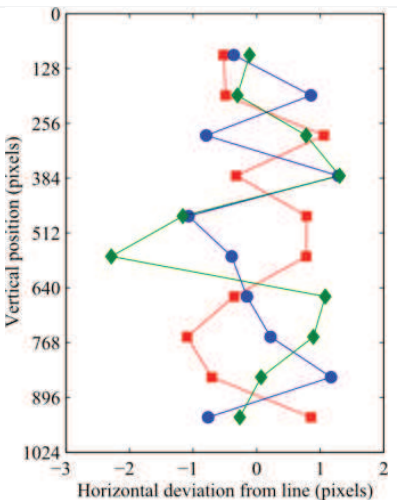

(b)

Fig. 10: Evaluation of distortion within optical microscope: (a) Identification of ruling boundaries, (b) Deviation of selected ruling boundaries from line of best fit.

correction. It is therefore expected that errors caused by distortion will be negligible in comparison to noise. To verify this, the image processing algorithm was run repeatedly over a single frame with limited vertical windows, separating the frame into ten bands. This would allow any geometric distortion to be observed in the curvature of these edges. The edges identified are shown in Fig. 10a, together with the deviation of three selected edge boundaries from the line of best fit in Fig. 10b. No considerable trends are visible confirming that distortion is unlikely to be a large issue. The variations in each plot are likely to be due to artefacts within the image. If distortion was found to play a significant role, fitted curves from Fig. 10b would allow the image to be corrected before applying the algorithm.

\subsubsection{Errors during Experimentation}

The use of the arc-length approximation (11) carries two errors. The first of these is due to using a linear approximation, which is:

$$
\Delta \theta_{V, \text { approx }}=\frac{d_{V}}{R_{V}}-\arctan \frac{d_{V}}{R_{V}} \approx \frac{1}{3} \theta_{V}^{3}
$$

Secondly, as the stage rotates, the rulings will rotate by the same angle. This process will continuously modify the scale. As the total rotation is the sum of the displacements between each frame, the output of the vision-based measurement system can be written as:

$$
\theta_{V}(t)=\frac{1}{R_{V}} \sum_{i=t_{0}}^{t} r\left(\theta_{t}[i-1]\right) \Delta d_{\mathrm{pix}}[i]
$$

where $\theta_{t}$ is the true stage rotation, $\Delta d_{\text {pix }}$ is the displacement between frames and $r$ is the variable image scale. Similar to (13), the scale will vary by a factor of $\sec \theta_{t}$, and hence $\theta_{V}$ will overestimate $\theta_{t}$. In rotating the stage monotonically from zero to an angle $\theta_{f}$, the greatest error will be when $\Delta d_{\text {pix }}$ is small, as the scale is less erroneous for smaller angles, hence large steps will carry less error. In the limit as the step size becomes infinitesimal, this error becomes: 


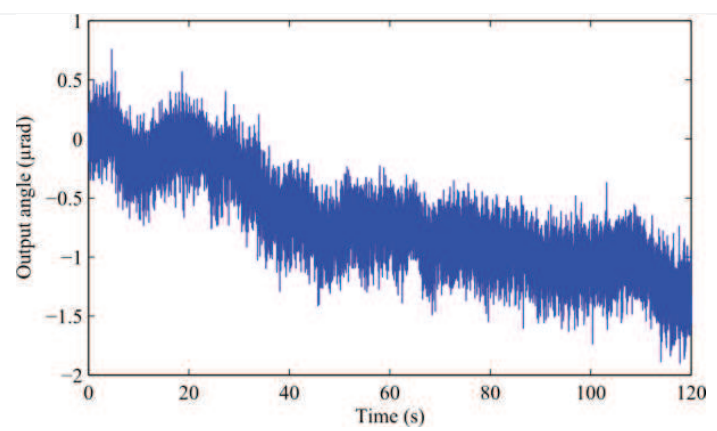

(a)

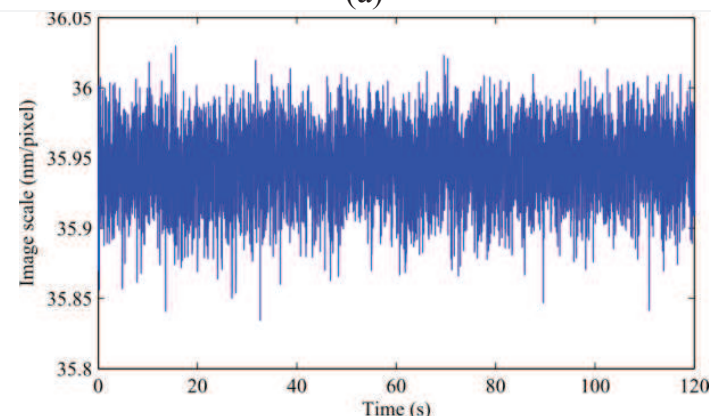

(b)

Fig. 11: Variation in image-based angular measurement output with the target slide fixed: (a) drift, (b) changes in image scale.

$$
\begin{aligned}
\Delta \theta_{V, \text { rot }} & =\frac{1}{R_{V}} \int_{\theta_{t}=0}^{\theta_{f}} r_{\text {ideal }}\left[\sec \theta_{t}-1\right]\left(\frac{R_{V}}{r_{\text {ideal }}} \mathrm{d} \theta_{t}\right) \\
& =\left[-\theta_{t}-\ln \left(\frac{\cos \frac{\theta_{t}}{2}-\sin \frac{\theta_{t}}{2}}{\cos \frac{\theta_{t}}{2}+\sin \frac{\theta_{t}}{2}}\right)\right]_{\theta_{t}=0}^{\theta_{f}} \\
& \approx \frac{\theta_{V}^{3}}{6}
\end{aligned}
$$

Drift of the output has been observed whilst the target slide was held stationary, as shown over a $120 \mathrm{~s}$ period in Fig. 11a. It is likely that this effect is due to creep within the mounting for the microscope, which was found to persist for long periods after the initial alignment. In this analysis the drift has been treated as an uncertainty in the final measurement, which increases linearly with time. The drift was repeatedly measured for $120 \mathrm{~s}$ ten times, and was found to have an RMS value of $1.19 \mu$ rad at the final time.

Due to (16) being the repeated summation of the inter-frame displacements, uncertainty in the image scale causes uncertainty in the final output. The variation in the image scale whilst the slide was stationary is shown in Fig. 11b. The RMS value of the variation in the scale was 24 pm/pixel. This uncertainty, which will accumulate over time can be predicted using (18).

$$
\Delta \theta_{V, \text { scale }}=\frac{1}{R_{V}} \sqrt{\sum_{i=t_{0}}^{t}\left(\Delta r[i] \Delta d_{\mathrm{pix}}[i]\right)^{2}}
$$

It is assumed that the uncertainty in the image scale is uncorrelated, as correlated deviations in the scale would indicate out-of-plane motion and a necessary change in scale. It is desirable to have (18) in a 
form dependent on the output quantities. By approximating the inter-frame displacement using the known angular velocity, (18) can be re-expressed as:

$$
\Delta \theta_{V, \text { scale }} \approx \frac{\Delta r}{r f_{V}} \sqrt{\sum_{i=t_{0}}^{t}(\dot{\theta}[i])^{2}},
$$

where $f_{V}$ is the frame rate of the image processing and it has been assumed that the overall change in the image scale is small. Due to the differing rates between the image processing and control processes, and data being collected only at the end of each control period, it is more appropriate to use the data available at the slower rate within the summation. Considering the difference in the number of terms in the summation at the vision system frequency compared to the control frequency, the uncertainty due to fluctuations in image scale can therefore be approximated using (20).

$$
\Delta \theta_{V, \text { scale }} \approx \frac{\Delta r}{r \sqrt{f_{V} f_{C}}} \sqrt{\sum_{j=t_{0}}^{t}(\dot{\theta}[j])^{2}}
$$

where $f_{C}$ is the slower control frequency, and $j$ denotes summation over the data collected at the control frequency.

Variations in pixel size may also result in uncertainty in the image scale. For the five rulings identified in Fig. 3, the standard deviation in inter-ruling spacing was 0.185 pixels, indicating that the pixel size in the horizontal direction is uniform. This corresponds to an uncertainty in scale of $47 \mathrm{pm}$, which is of a similar magnitude to the RMS fluctuation in the scale considered above. It should be noted that the scale is calculated from the pixels towards the centre of the image; hence the effect of variations in pixel sizing will be further reduced.

Artefacts on the edge of a ruling, where it fails to be perfectly straight, such as those seen on the far right ruling in the frame depicted in Fig. 3, will cause uncertainty in the location of the ruling's centre. In the experimentation conducted, this ruling was one of the worst cases observed. Due to the vertical averaging of the pixels in the algorithm, the effect of these artefacts is reduced. When the edge detection was run on the ruling edge with the artefacts excluded from the averaging step, a difference in the identified edge position of 0.14 pixels was calculated. Hence, it is estimated that such artefacts will introduce an uncertainty of $0.05 \mu \mathrm{rad}$.

Similar to the interferometry-based measurement, data-age will also impact the final output. The output uncertainty is therefore determined by (10). This uncertainty will be smaller for the vision-based system as it updates more frequently and has a low angular velocity. Finally, vibrations and environmental noise also introduce uncertainty, which has again been found as the variation in the system output for a fixed stage, over a short timeframe to discount drift. 


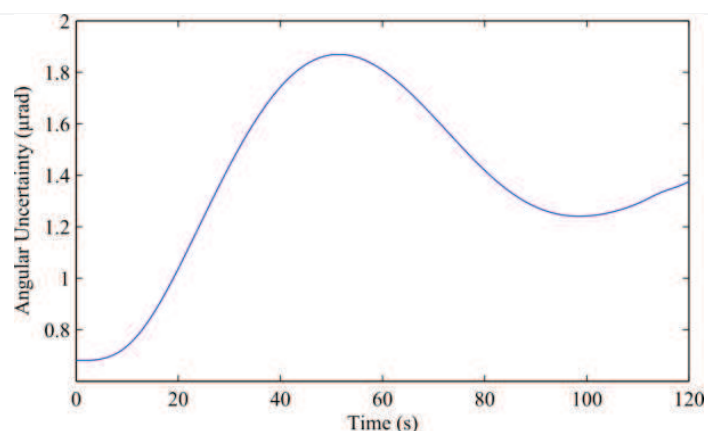

(a)

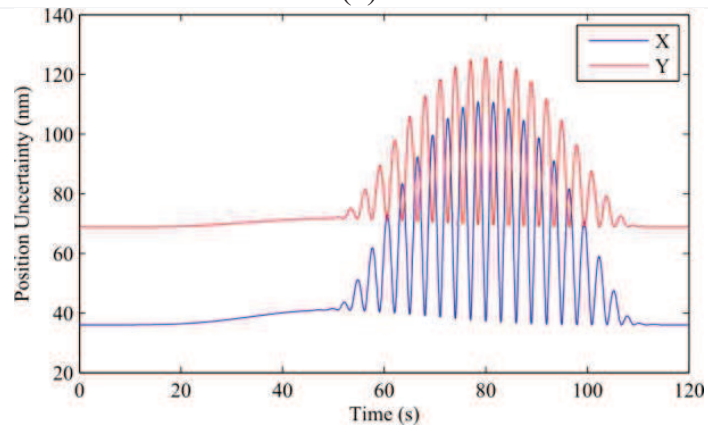

(b)

Fig. 12: Estimation of uncertainties in final outputs during experimentation: (a) $\theta$, (b) $X$ and $Y$.

\subsection{Propagation of Individual Sources}

From the uncertainty within each sensor, various transformations that are applied to the individual outputs will also impact on the uncertainty in the final position estimates. As shown in Fig. 7, these include the addition of sensor outputs to find $\theta$ and also the rotation of interferometer measurements to find the $X$ and $Y$ outputs.

The centre of the coordinate origin is displaced from the axis of rotation of the stage by an unknown amount (the concentricity), which couples an uncertainty to the $X$ and $Y$ outputs. As a rotation is introduced, the origin of the coordinate system on the mechanism base will move along a circular arc (in the $X-Y$ plane) about the axis of rotation. For the stage utilised, this concentricity is specified to be less than $25 \mu \mathrm{m}$ (assumed to be uniformly distributed). As the displacement of the origin from the centre of rotation is uncertain, it must be assumed that this uncertainty is added to each of the linear outputs to its full extent. This can therefore be approximated as:

$$
\Delta x_{i, \text { concen }}=r_{\text {concen }} \theta_{V}
$$

The maximum standard uncertainty due to concentricity is therefore approximately $19.5 \mathrm{~nm}$.

The rotation of the interferometer measurements to find the $X$ and $Y$ outputs is given by:

$$
\left[\begin{array}{l}
x \\
y
\end{array}\right]=\left[\begin{array}{cc}
\cos \theta & -\sin \theta \\
\sin \theta & \cos \theta
\end{array}\right]^{T}\left[\begin{array}{l}
x_{i} \\
y_{i}
\end{array}\right]
$$

Hence, the combined uncertainties in the outputs can be found through (23) and (24). 


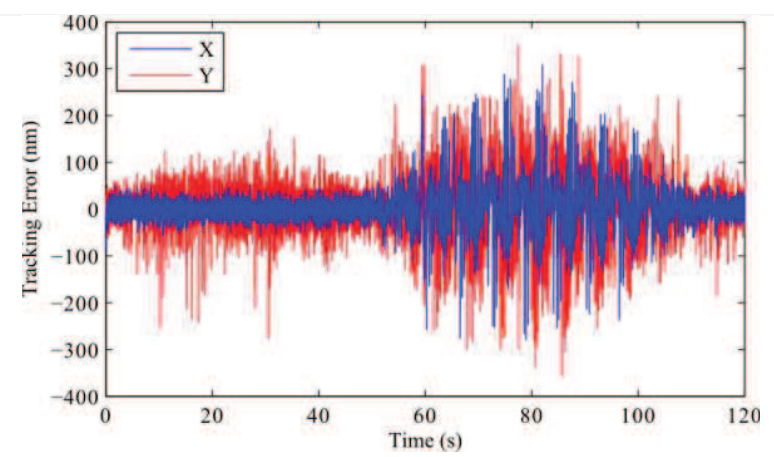

(a)

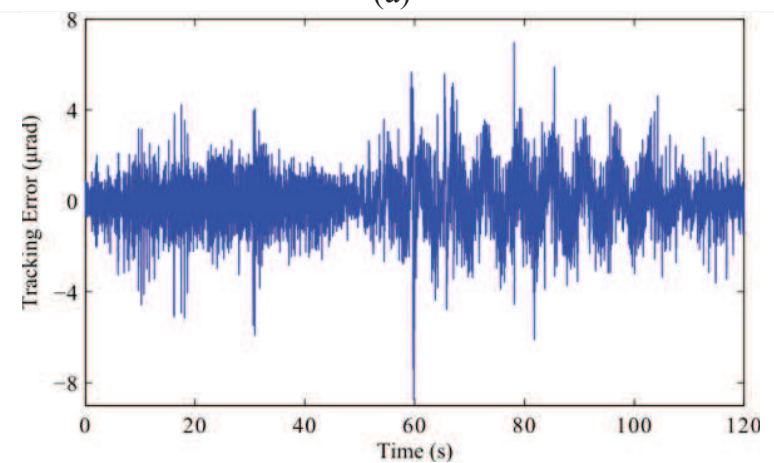

(b)

Fig. 13: Experimental control tracking error: (a) $X$ and $Y$, (b) $\theta$.

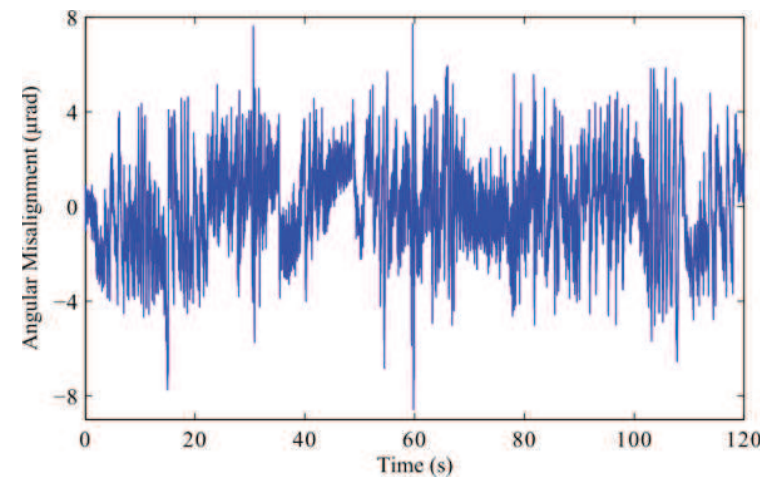

Fig. 14: Misalignment of laser system measured during experimentation.

$$
\begin{aligned}
(\Delta x)^{2} & =\left(\Delta x_{i} \cos \theta\right)^{2}+\left(\Delta y_{i} \sin \theta\right)^{2}+\left(\left(-x_{i} \sin \theta+y_{i} \cos \theta\right) \Delta \theta\right)^{2} \\
& \approx\left(\Delta x_{i}\right)^{2}+\left(\theta \Delta y_{i}\right)^{2}+\left(\left(y_{i}-x_{i} \theta\right) \Delta \theta\right)^{2} \\
(\Delta y)^{2} & =\left(\Delta x_{i} \sin \theta\right)^{2}+\left(\Delta y_{i} \cos \theta\right)^{2}+\left(\left(-x_{i} \cos \theta-y_{i} \sin \theta\right) \Delta \theta\right)^{2} \\
& \approx\left(\theta \Delta x_{i}\right)^{2}+\left(\Delta y_{i}\right)^{2}+\left(\left(x_{i}+y_{i} \theta\right) \Delta \theta\right)^{2}
\end{aligned}
$$

Fig. 12 shows the predicted uncertainty in measurements throughout the trajectory of Section 2.2, where sources of uncertainty have been added in quadrature.

\section{Experimental Results and Discussion}

Fig. 13 shows the tracking errors observed during experimentation with closed loop control. The misalignment, critical for geometric errors, is shown in Fig. 14. Their magnitudes are summarised in Table 4. 
Table 4: Summary of experimental tracking errors and optical misalignment

\begin{tabular}{ccc}
\hline \hline & RMS & Maximum \\
\hline$X$ & $41.7 \mathrm{~nm}$ & $309 \mathrm{~nm}$ \\
$Y$ & $67.4 \mathrm{~nm}$ & $357 \mathrm{~nm}$ \\
$\theta$ & $1.16 \mu \mathrm{rad}$ & $8.74 \mu \mathrm{rad}$ \\
\hline$\theta_{e}$ & $1.96 \mu \mathrm{rad}$ & $8.58 \mu \mathrm{rad}$ \\
\hline \hline
\end{tabular}

These figures show the deviation of the controlled output from the estimate of the position provided by the measurement systems. The bounds on the true tracking performance are therefore the summation of the measurement uncertainty with the control tracking error. Improvements to this performance can therefore be made in two ways: refinements in the apparatus and sensing technique to reduce measurement uncertainties, and the establishment of more advanced controllers to reduce the control tracking error.

\subsection{Control Tracking Errors}

As can be seen from Fig. 13a, tracking errors along the $Y$ direction were larger than those in $X$. Due to the larger offset between the $Y$ axis and the measurement axis of the HSPMI, this was caused by Abbe errors, and the increased fluctuation of the $Y$ measurement due to angular misalignment. Moreover, for this measurement technique, it can be seen that Abbe errors allowed the misalignment error to couple into position measurement.

Despite the remarks in Section 4.2.1 regarding the inability to correct accurately for such Abbe errors, it was observed that tracking errors were reduced to some extent through the compensation of $y_{i}$ given by (25).

$$
y_{i}^{\prime}=y_{i}-\eta \theta_{e}
$$

Through finding the parameter that minimised the RMS error during the first $35 \mathrm{~s}$ (where there was no desired $Y$ motion but the misalignment controller was functioning), it was found that the parameter $\eta$ was not the axis displacement $b(30 \mathrm{~mm})$ in (9), but instead $9.20 \mathrm{~mm}$. This compensation was utilised for the controller results presented in this paper.

The sampling rate utilised for control was limited by the speed of the slowest sensor, being the laser interferometry-based sensing and measurement system. This impacted upon the measurement uncertainty, which then affected the tracking errors. Ultimately, this manifested as a data age uncertainty, which was compounded by the unknown age of the interferometer sample within the previous period. Hence, the error shown in Fig. 13 was actually the tracking error coupled with the data age uncertainty. The error observed therefore increased with the speed along each measurement axis,and Fig. 13 clearly demonstrates this phenomenon. Performance will be improved through the 
increase in sampling rate (feasible through the use of a faster interface to the interferometer controller, such as a VME bus), or through the reduction of the trajectory's velocity.

\subsection{Uncertainties}

The largest relative uncertainties were observed in the measurements of $X$ and $Y$ motions, dominated by the uncertainty in interferometer measurements. In this case, the uncertainties when the mechanism was stationary were $0.07 \%(X)$ and $0.13 \%(Y)$ of the range of motion of the trajectory. This uncertainty was only $0.05 \%$ for $\theta$ measurement. Assuming that the tracking error was also uncertain, the combined standard uncertainties at the final time (calculated over a $5 \mathrm{~s}$ period) were $39.1 \mathrm{~nm}, 76.8 \mathrm{~nm}$, and $1.52 \mu \mathrm{rad}$ for $X, Y$, and $\theta$ respectively. The maximum combined standard uncertainties in all three axes occurred during the fastest motion in the $X$ and $Y$ axes (at approximately $80 \mathrm{~s}$ ), which were $134 \mathrm{~nm}$, $164 \mathrm{~nm}$ and $2.22 \mu \mathrm{rad}$.

As shown in Fig. 11a, drift in the vision-based angular measurement was observed to increase approximately linearly, where short term fluctuations were likely to have resulted from external vibrations. Over repeated trials, this drift was seen to be unidirectional, and its magnitude could be predicted. Whilst the drift was treated as an uncertainty in this study, it therefore would be possible to provide compensation for the drift in future applications.

Throughout this analysis it has been assumed that the error in the raw HSPMI measurement is negligible following its factory calibration. Similarly, the calibration of the misalignment and visionbased angular measurement systems against the HSPMI angular axis has been assumed to carry an uncertainty which is negligible in comparison to the environmental noise sources. Uncertainty in the calibration device would therefore need to be taken into consideration if this were found not to be true, or if the other sources of uncertainty have been minimised to a significant extent.

From Table 2 it can be seen that the largest sources of HSPMI uncertainty result from the data age uncertainty, Abbe uncertainty, and changes in environmental conditions. Consideration of each contribution during system design is therefore the simplest method to reduce their magnitude. Variations in the refractive index of the optical path can similarly be reduced through tighter control of the environment, such as the use of an enclosure.

Abbe uncertainty can be reduced easily by proper alignment of the measurement axes with the mechanism's coordinate axes. In the case of this methodology, the PSD misalignment sensor and the $Y$ axis HSPMI sharing the same reflector necessitated the use of the displaced axis. Future redesigns of the system should therefore shift the PSD axis away from its current position.

There is difficulty in reducing the uncertainty due to concentricity, seen in Fig. 12b as the variation of the linear uncertainties with angular position. The use of a more precise rotation stage may be mandated 
in this case. However, it may be possible to locate the actual rotation centre by measuring the change in the linear axes in response to a pure rotation, and later compensating for this effect.

\section{Conclusion}

In this paper, the errors and uncertainties associated with the measurement methodology for coupled angular and linear motions have been analysed. This has been explored through the experimental investigation of the closed-loop tracking performance of a 3-DOF flexure-based mechanism. Whilst controller tracking errors are $41.7 \mathrm{~nm}, 67.4 \mathrm{~nm}$, and $1.16 \mu \mathrm{rad}(\mathrm{RMS})$ for $X, Y$, and $\theta$ respectively, due to measurement uncertainties, the tracking performance can only be stated to have been achieved within $118 \mathrm{~nm}, 143 \mathrm{~nm}$, and $2.20 \mu \mathrm{rad}$ (RMS). It is seen that for such a methodology, some sources of error within the sensing systems have a direct impact upon the performance of the control system. In particular, these include data age errors and Abbe errors which affect the performance of the laser interferometry-based linear axis measurements.

\section{Acknowledgements}

This work was supported by ARC LIEF (Grants LE0347024 and LE0775692), and ARC Discovery Projects (Grants DP0986814, DP110104970, and DP140104019).

\section{References}

[1] K-B Choi, JJ Lee, Passive compliant wafer stage for single-step nano-imprint lithography, Review of Scientific Instruments, 76(2005) 075106.

[2] M Holmes, R Hocken, D Trumper, The long-range scanning stage: a novel platform for scannedprobe microscopy, Precision Engineering, 24(2000) 191-209.

[3] A Ferreira, C Cassier, S Hirai, Automatic microassembly system assisted by vision servoing and virtual reality, IEEE/ASME Transactions on Mechatronics, 9(2004) 321-33.

[4] MNM Zubir, B Shirinzadeh, Y Tian, Development of a novel flexure-based microgripper for high precision micro-object manipulation, Sensors and Actuators A: Physical, 150(2009) 257-66.

[5] Y Tian, D Zhang, B Shirinzadeh, Dynamic modelling of a flexure-based mechanism for ultraprecision grinding operation, Precision Engineering, 35(2011) 554-65.

[6] BJ Kenton, KK Leang, Design and control of a three-axis serial-kinematic high-bandwidth nanopositioner, IEEE/ASME Transactions on Mechatronics, 17(2012) 356-69.

[7] Y Li, Q Xu, A totally decoupled piezo-driven XYZ flexure parallel micropositioning stage for micro/nanomanipulation, IEEE Transactions on Automation Science and Engineering, 8(2011) 265-79.

[8] S Polit, J Dong, Development of a high-bandwidth XY nanopositioning stage for high-rate micro/nanomanufacturing, IEEE/ASME Transactions on Mechatronics, 16(2011) 724-33.

[9] Y Tian, B Shirinzadeh, D Zhang, A flexure-based five-bar mechanism for micro/nano manipulation, Sensors and Actuators A: Physical, 153(2009) 96-104. 
[10] Y-M Choi, JJ Kim, J Kim, D-G Gweon, Design and control of a nanoprecision XYA scanner, Review of Scientific Instruments, 79(2008) 045109.

[11] H Wang, X Zhang, Input coupling analysis and optimal design of a 3-DOF compliant micropositioning stage, Mechanism and Machine Theory, 43(2008) 400-10.

[12] Y Tian, B Shirinzadeh, D Zhang, Y Zhong, Three flexure hinges for compliant mechanism designs based on dimensionless graph analysis, Precision Engineering, 34(2010) 92-100.

[13] HC Liaw, B Shirinzadeh, J Smith, Robust neural network motion tracking control of piezoelectric actuation systems for micro/nanomanipulation, IEEE Transactions on Neural Networks, 20(2009) 356-67.

[14] HC Liaw, B Shirinzadeh, Constrained motion tracking control of piezo-actuated flexure-based four-bar mechanisms for micro/nano manipulation, IEEE Transactions on Automation Science and Engineering, 7(2010) 699-705.

[15] U Bhagat, B Shirinzadeh, Y Tian, Experimental study of laser interferometry based motion tracking of a flexure-based mechanism, International Journal of Intelligent Mechatronics and Robotics, 1(2011) 31-45.

[16] HC Liaw, B Shirinzadeh, Robust adaptive constrained motion tracking control of piezo-actuated flexure-based mechanisms for micro/nano manipulation, IEEE Transactions on Industrial Electronics, 58(2011) 1406-15.

[17] M Al Janaideh, S Rakheja, C-Y Su, An analytical generalized Prandtl-Ishlinskii model inversion for hysteresis compensation in micropositioning control, IEEE/ASME Transactions on Mechatronics, 16(2011) 734-44.

[18] J Minase, T-F Lu, B Cazzolato, S Grainger, A review, supported by experimental results, of voltage, charge and capacitor insertion method for driving piezoelectric actuators, Precision Engineering, 34(2010) 692-700.

[19] Y Qin, B Shirinzadeh, Y Tian, D Zhang, Design issues in a decoupled XY stage: Static and dynamics modeling, hysteresis compensation, and tracking control, Sensors and Actuators A: Physical, 194(2013) 95-105.

[20] GA Al-Kindi, B Shirinzadeh, An evaluation of surface roughness parameters measurement using vision-based data, International Journal of Machine Tools and Manufacture, 47(2007) 697-708.

[21] DJ Cappelleri, G Piazza, A two dimensional vision-based force sensor for microrobotic applications, Sensors and Actuators A: Physical, 171(2011) 340-51.

[22] U Bhagat, B Shirinzadeh, Y Tian, D Zhang, Experimental analysis of laser interferometry-based robust motion tracking control of a flexure-based mechanism, IEEE Transactions on Automation Science and Engineering, 10(2013) 267-75.

[23] J Hrabina, J Lazar, P Klapetek, O Číp, Multidimensional interferometric tool for the local probe microscopy nanometrology, Measurement Science and Technology, 22(2011) 094030. 
[24] J Lazar, P Klapetek, O Č́íp, M Čížek, M Šerý, Local probe microscopy with interferometric monitoring of the stage nanopositioning, Measurement Science and Technology, 20(2009) 084007.

[25] H-C Yeh, W-T Ni, S-S Pan, Real-time motion control With subnanometer heterodyne interferometry, International Journal of Modern Physics D, 11(2002) 1087-99.

[26] L Clark, B Shirinzadeh, Y Tian, D Oetomo, Laser-based sensing, measurement and misalignment control of coupled linear and angular motion for ultra-high precision movement, IEEE/ASME Transactions on Mechatronics, (Accepted), DOI: 10.1109/TMECH.2014.2301824.

[27] Y Tian, B Shirinzadeh, D Zhang, Design and dynamics of a 3-DOF flexure-based parallel mechanism for micro/nano manipulation, Microelectronic Engineering, 87(2010) 230-41.

[28] L Clark, B Shirinzadeh, U Bhagat, J Smith, A vision-based measurement algorithm for micro/nano manipulation, 2013 IEEE/ASME International Conference on Advanced Intelligent Mechatronics, IEEE, Wollongong, Australia, 2013, pp. 100-5.

[29] Y Tian, B Shirinzadeh, D Zhang, X Liu, D Chetwynd, Design and forward kinematics of the compliant micro-manipulator with lever mechanisms, Precision Engineering, 33(2009) 466-75.

[30] WJ Zhang, J Zou, LG Watson, W Zhao, GH Zong, SS Bi, The constant-jacobian method for kinematics of a three-DOF planar micro-motion stage, Journal of Robotic Systems, 19(2002) 6372.

[31] T-F Lu, DC Handley, YK Yong, C Eales, A three-DOF compliant micromotion stage with flexure hinges, Industrial Robot, 31(2004) 355-61.

[32] Y Qin, Y Tian, D Zhang, B Shirinzadeh, S Fatikow, A novel direct inverse modeling approach for hysteresis compensation of piezoelectric actuator in feedforward applications, IEEE/ASME Transactions on Mechatronics, 18(2013) 981-9.

[33] KP Birch, MJ Downs, Correction to the updated Edlen equation for the refractive index of air, Metrologia, 31(1994) 8-10. 